\title{
5 Ethanol-guided behavior in Drosophila larvae
}

Isabell Schumann', Michael Berger'2, Nadine Nowag', Yannick Schäfer², Juliane Saumweber ${ }^{1}$, Henrike Scholz ${ }^{2}$ and Andreas S. Thum ${ }^{1^{*}}$

1Department of Genetics, University of Leipzig, 04103 Leipzig, Germany;

${ }^{2}$ Department of Biology, University of Cologne, D-50674 Cologne, Germany

*Correspondence: Andreas S. Thum, Faculty of Life Sciences, Institute of Biology, Department of Genetics, University of Leipzig, Talstraße 33, 04103 Leipzig, Germany

E-mail: andreas.thum@uni-leipzig.de

15

Running head: Ethanol responses of Drosophila larvae

Keywords: Drosophila larvae, choice behavior, chemosensation, survival, reward, learning and memory 


\section{Abstract}

Chemosensory signals allow vertebrates and invertebrates not only to orient in its environment toward energy-rich food sources to maintain nutrition but also to avoid unpleasant or even poisonous substrates. Ethanol is a substance found in the natural environment of Drosophila melanogaster. Accordingly, D. melanogaster has evolved specific sensory systems, physiological adaptations, and associated behaviors at its larval and adult stage to perceive and process ethanol.

To systematically analyze how $D$. melanogaster larvae respond to naturally occurring ethanol, we examined ethanol-induced behavior in great detail by parametrically reevaluating existing approaches and comparing them with new experiments. Using behavioral assays, we confirm that larvae are attracted to different concentrations of ethanol in their environment. This behavior is controlled both by olfactory and contact cues. It is independent of previous exposure to ethanol in their food. Moreover, moderate, naturally occurring ethanol concentration of $4 \%$ results in increased larval fitness. On the contrary, higher concentrations of $10 \%$ and $20 \%$ ethanol, which rarely or never appear in nature, increase larval mortality. Finally, ethanol also serves as a positive teaching signal in learning and memory and updates valence associated with simultaneously processed odor information.

Since information on how larvae perceive and process ethanol at the genetic and neuronal level is limited, the establishment of standardized assays described here is an important step towards their discovery. 


\section{Introduction}

Communication with the environment through chemical signals is an essential process for the survival of most if not all organisms. Specialized signal transduction pathways are used to detect chemical cues and convert information into neuronal activity that induces appropriate behavioral output, which is evolutionary conserved between invertebrates and vertebrates, identified by biochemical, molecular and genetic approaches ${ }^{1}$. Important insights into principals of chemosensory perception and information processing are provided by genetically modifiable organisms such as the fruit fly $D$. melanogaster ${ }^{2-5}$. This includes also the larval central nervous system with its simpler structure consisting of only about 10,000 neurons, but equally provides access to combinations of genetic tools, robust behavioral assays, the possibility of transgenic single-cell manipulation, and even connectome data of the central nervous system ${ }^{6-14}$. Various studies have identified chemosensory stimuli that larvae perceive from their environment. Most odorants, are attractive to larvae in a dose-dependent manner 15-17. Likewise, larvae show dose-dependent responses to gustatory cues ${ }^{18-}$ 22. Even the characteristics of the substrate seem to influence larval chemosensory responses ${ }^{23,24}$. However, to understand how larvae orient in their complex chemosensory environment, further studies are needed on additional environmental occurring stimuli, such as ethanol.

Most Drosophila species are saprophagous and feed on decaying sweet substrates like rotting fruits, which contain ethanol produced by natural fermentation ${ }^{25}$. Adult flies exhibit acute ethanol responses similar to those of mammals: as ethanol concentration increases, flies exhibit locomotor stimulation, loss of postural control, and eventually sedation ${ }^{26}$. With repeated exposure, adults develop tolerance to the effects of ethanol 27. It is even assumed that $D$. melanogaster has altered its ecological niche to benefit from food sources characterized by a higher alcohol concentration ${ }^{28}$. D. melanogaster has an unusually high alcohol dehydrogenase (Adh) activity within its genus, which allows to deal with higher ethanol levels to occupy microhabitats that are not accessible to other species such as Drosophila simulans, which have a lower Adh activity ${ }^{29}$. In addition, larvae are even able to perceive and selectively consume food containing ethanol when they are infected by parasitic wasps ${ }^{30}$. A higher ethanol in the larval hemocoel due to increased consumption of ethanol enriched food leads to enhanced death of the growing parasites and thus increases the larval survival rate. This means that $D$. melanogaster larvae can adjust their alcohol consumption depending on the particular situation, and use it as a kind of medical treatment. Accordingly, ethanol is an important ecological parameter, which has selected to the establishment of specific larval sensory systems, physiological adaptations and related behaviors.

Therefore, it is plausible that several studies have been able to show that $D$. melanogaster larvae preferentially migrate to substrates that contain ethanol. For instance, different wild-type strains derived from Australian populations revealed strong preferences for $6 \%$ ethanol ${ }^{31-33}$. Even $17 \%$ ethanol was attractive for two larval 
strains that were either homozygous for the $A d h^{F}$ or the $A_{d h} S$ allele ${ }^{34} . A d h^{F}$ and $A d h^{S}$ describe two functional allozymes that exist in natural populations of Drosophila species on several continents ${ }^{35,36}$. The polymorphism is maintained by natural selection; while $\mathrm{Adh}^{\mathrm{F}}$ is showing a higher enzymatic activity for ethanol, $\mathrm{Adh}^{\mathrm{S}}$ is more resistant to higher environmental temperatures ${ }^{37}$. However, there are also studies in which ethanol is not attractive to larvae ${ }^{15,38,39}$, which complicates the interpretation of the various results. Therefore, we parametrically analyzed ethanol guided behavior by combining published and novel experimental designs.

In recent years, a set of well-defined larval behavioral assays was established to investigate substrate choice, feeding, survival, and associative learning and memory $14,18,19$. It is therefore possible to analyze how larvae react to individual chemical components of its environment. Fructose, for example, provides nutrition and acts as a positive teaching signal during learning ${ }^{20,21}$. On the other hand, many chemicals that

100 humans categorize as bitter are also repulsive to the larvae (for example among others caffeine, denatonium, or quinine) and can act as a negative teaching signal (caffeine, quinine) $18,19,22,40$. Sodium chloride even proved to be dichotomous, being attractive and a positive teaching signal at low concentrations and repellent and a negative teaching signal at high concentrations ${ }^{41,42}$. Using a set of standardized assays, we

105 have now addressed the effect of ethanol on larval behavior. Our results support previous studies suggesting that the odorant ethanol is attractive to larvae in a doses dependent manner at moderate naturally occurring concentrations and has a positive effect on larval survival. The larval ethanol choice is based on smell and taste. Finally, ethanol can be used in associative olfactory learning paradigms to establish appetitive

110 memories as it provides a positive teaching signals.

\section{Results}

\section{Drosophila melanogaster larvae are attracted to ethanol}

Several studies have repeatedly shown that $D$. melanogaster larvae are attracted to ethanol ${ }^{31-34}$. However, in almost all studies there are slight differences in the applied assays, used substrates and mode of ethanol presentation. This might influence the observed results. In some cases ethanol is attractive and in other not 15,38,39. Therefore, we first reanalyzed the attractiveness of ethanol in a widely applied standardized assay. We analyzed the preference of wild-type Canton-S to ethanol by observing their approach behavior to a substrate that contains different concentration of ethanol (Fig. 1). The ethanol concentration ranged from $1 \%$ to $30 \%$. We found that Canton-S and $w^{1118}$ larvae are attracted to ethanol in a dose-dependent manner (Fig. 1b). The attractiveness of ethanol peaked at around $4-10 \%$ ethanol for Canton-S. For $10 \%$ and $20 \%$ ethanol Canton-S-larvae showed higher preferences than $w^{1118}$ larvae. Additionally, we observed the attractiveness to $8 \%$ ethanol of the Canton-S larvae for $120 \mathrm{~min}$ (Fig. 1c). The attraction to ethanol remained equally stable and no sedative 
effect on larval locomotion was seen during this prolonged ethanol exposure. To investigate the influence of ethanol pre-exposure on the substrate choice of larvae we compared how animals reared on standard food (containing $1 \%$ ethanol) versus animals reared for one or two generations on ethanol-free food respond to $8 \%$ ethanol (Fig. 1d). Canton-S larvae grown for one or two generations on ethanol-free food preferred the ethanol containing site similar to larvae raised on standard food. These results show that $D$. melanogaster larvae are attracted to ethanol in a concentrationdependent manner. Pre-exposure to ethanol and the duration of the test period do not alter the attraction.

\section{Ethanol attraction changes during post-embryonic development}

The life cycle of $D$. melanogaster comprises three larval stages (L1, L2 and L3), clearly separated by two molting stages, which occur approximately 48 and $72 \mathrm{~h}$ after egg laying (AEL). To understand whether ethanol choice changes during larval development, we analyzed eight groups of Canton-S larvae from 36 - $120 \mathrm{~h}$ AEL every

$14512 \mathrm{~h}$ for their $8 \%$ ethanol attraction (Fig. 2). We chose this concentration because it was most preferred by the larvae in the first experiment (Fig. 1). Each of the tested groups showed an attraction to ethanol (Tab. 1). However, during the first and second molt, the preferences are reduced (Fig. 2, Tab. 1). Furthermore, in L3 larvae the preference decreases with age.

\section{Ethanol has nutritional benefit for the larva}

Ethanol can be used as energy source (reviewed in ${ }^{43}$ ). However, the nutrient gain for the larva appears to be lower, as survival rates on ethanol substrates are lower than those of adult Drosophila ${ }^{44,45}$ and also than those of larvae on sugar diets 20 . To complicate matters, ethanol is pharmacologically active and in higher concentrations toxic and needs to be neutralized ${ }^{46-48}$. These diametrically opposed effects complicate the interpretation of ethanol-dependent survival studies. To resolve and better interpret these effects, we analyzed ethanol dependent survival under standard conditions applied for other environmental cues ${ }^{18,20,23}$. We put L2 Canton-S larvae in food vials containing $1 \%$ agarose an either no ethanol, $4 \%$, $8 \%$, or $20 \%$ (Fig. 3). We calculated a survival rate by counting the number of living larvae and pupae for eight days in a $24 \mathrm{~h}$ cycle (Fig. 3b, c). The results show two effects: at $4 \%$ ethanol, more larvae survive than on pure agarose until pupation (73.8\% versus $35.8 \%$; Fig. 3b, c, light blue line) and at higher concentration of $20 \%$ only few animals survive until pupation $(12.2 \%$; Fig. $3 b$, c, dark blue line). The larval survival rates on pure agarose and $8 \%$ ethanol are right in between (Fig. 3b, $44.9 \%$ versus $60.9 \%$, green versus blue line). However, of these animals less larvae pupated on $8 \%$ ethanol in comparison to pure agarose condition (Fig. 3c). 


\section{Larvae perceive ethanol as an olfactory cue}

Initially we performed substrate choice experiments that allowed larvae to get into direct contact with ethanol in addition to smelling the odorant source (Fig. 1, 2). To disentangle the olfactory from contact cues we refined our behavioral approach by presenting ethanol in custom-made Teflon containers with perforated lids. This eliminates a physical contact with the substrate to specifically address the olfactory response of larvae (Fig. 4). We compared the attraction of $8 \%$ ethanol to three wellknown attractive odorants: 1-octanol (1-OCT), amyl acetate (AM), and benzaldehyde

180 (BA) ${ }^{15-17}$. Canton-S larvae showed olfactory preference for all four odorants (Fig. 4b). Thus, $D$. melanogaster larvae perceive ethanol as an attractive olfactory stimulus. In the next step we analyzed whether the larva can detect other odorants in a homogeneous ethanol background, which is a basic requirement for further odorantethanol learning experiments (Fig. 4d). Since BA triggered the greatest behavioral 185 response, we used this stimulus to test whether larvae can still perceive it on 5\%, 8\%, $10 \%$ and $20 \%$ ethanol containing agarose plates (Fig. 4d). For all four ethanol concentrations wild-type Canton-S larvae were similarly attracted to BA (Fig. 4d). Therefore, in the assay $D$. melanogaster larvae could distinguish BA from a week or high concentrated ethanol background.

\section{Ethanol provides a teaching signal for larval olfactory learning}

Larvae of $D$. melanogaster are able to associate odorants (conditioned stimulus) with cues of different sensory modalities (unconditioned stimulus) to establish appetitive or

195 aversive olfactory memories. So far, tastants, temperature, vibration, electric shock, and light have been identified as teaching signals (reviewed in ${ }^{14}$ ). In contrast to adult flies, ethanol has not yet been tested as reinforcer. Therefore, we performed standardized learning experiments to validate the potential of ethanol in differential conditioning. In this assay larvae are trained three times with a given concentration of 200 ethanol $(2.5 \%, 8 \%$, or $20 \%$ as a teaching signal) and then tested on an agarose plate for their odorant preference between the previously ethanol paired and the non-paired odorant (Fig. 5). Higher ethanol concentrations of $8 \%$ and $20 \%$, in contrast to a lower concentration of $2.5 \%$, provide an appetitive teaching signal for larval olfactory learning (Fig. 5b). For appetitive olfactory learning with fructose, it has been reported 205 that larvae show no memory when tested in the presence of the unconditioned stimulus. This is probably because in the presence of food the larvae do not have to search for food 41,49 . This also seems to be the case for ethanol, since larvae trained with $8 \%$ ethanol only recall odor-ethanol memory on pure agarose, but not on a test plate containing $8 \%$ ethanol (Fig. 5c). Ethanol memory also appears to be somehow 210 similar to fructose memory, as even $2 \mathrm{M}$ fructose added to the test plate prevents the recall of the odor-ethanol memory. 


\section{Ethanol does not alter appetitive and aversive olfactory memory}

Treating larvae with $20 \%$ ethanol for 20 min impairs aversive odorant-heat shock memory after one-odor conditioning at $35^{\circ} \mathrm{C} 50$. The effect of ethanol was absent, when the odorant was paired with a higher temperature heat-shock of $41^{\circ} \mathrm{C}$, which likely forms a stronger memory unsusceptible for ethanol treatment. Therefore, we similarly investigated whether the 20 min incubation with 20\% ethanol interferes with larval olfactory memory formation when the odorant is paired with fructose or salt (Fig. 6). We found that both, aversive salt memory (1.5 M NaCl; Fig. 6a) and appetitive sugar memory (0.01 $\mathrm{M}$ fructose; Fig. 6b), showed no reduction after ethanol treatment in comparison to $\mathrm{H}_{2} \mathrm{O}$ treated larvae. Therefore, the olfactory memory reinforced by salt or fructose was not altered by ethanol treatment.

\section{Discussion}

Ethanol is an important stimulus in the environment of adult and larval $D$. melanogaster (reviewed in ${ }^{51,52}$ ). The concentration of ethanol in the natural habitat of this species

230 vary between $0.6 \%$ in ripe hanging fruits and up to $4.5 \%$ in rotting ones ${ }^{53}$. In some man-made environments, such as wine cellars, the ethanol concentration can reach more than $10 \%{ }^{53-55}$. Given that adult $D$. melanogaster often carry yeast to the egg laying sites and inoculate ripening fruits, the induced fermentation and associated ethanol production is likely to result in higher concentrations at the larval stage ${ }^{56}$.

235 Therefore, the naturally occurring range of ethanol concentrations experienced by a larva is in agreement with most of the concentrations tested in our behavioral analysis (Fig. 1, Fig.3, and Fig. 5). Only the highest concentration tested (20\% and 30\% ethanol) do not occur in the natural habitat of the larva. Of course, one has to keep in mind that our experiments were performed under laboratory conditions using a

240 substrate of agarose mixed with ethanol. Due to the evaporation of ethanol, which is indeed volatile, especially higher ethanol concentrations could have been lower in reality. However, these effects are likely to be small, as studies have shown that within the first few hours, ethanol levels drop only slightly in a $13 \%$ ethanol containing Petri dish ${ }^{55}$. This corresponds approximately to the period of preparation of the plates and

245 the conduction of the ethanol attraction and learning experiments. Similarly, evaporation seems to be of little relevance in the survival experiments, where the experimental vials were changed every day. It was shown that the ethanol concentration in vials closed with polyurethane bungs remains very stable over several days (in bottles with $6 \%$ ethanol in the medium the fall is to $3.5 \%$ after six days) ${ }^{55}$.

\section{The ethanol attraction is based on various sensory modalities}

We show that $D$. melanogaster larvae preferentially migrate to substrates containing ethanol, regardless of whether they have been previously exposed to ethanol or not. Larval attraction appears to be doses-dependent reaching a plateau between $4 \%$ and $10 \%$ (Fig.1 and Fig. 2). Our results are in agreement with several published findings 
that have shown that larvae prefer $1 \%{ }^{57}, 2.5 \%{ }^{58}, 5 \%{ }^{58}, 6 \%^{31-33}, 10 \%{ }^{57,58}, 17 \%{ }^{34}$, $20 \%{ }^{58}$ and even pure ethanol ${ }^{57}$. Thus, our standardized experimental design provides a robust behavioral assay that allows to identify the neural and molecular basis of larval ethanol substrate choice. However, it must be mentioned that there are some studies on larval ethanol substrate choice, where the tested animals show no or only weak attraction to ethanol concentrations from $2 \%$ to $6 \% 38,39,59$ and even aversion at higher concentrations 39,59. However, it is not clear whether, in addition to methodological matters such as a low volume of applied ethanol ${ }^{38}$, other factors such as the enzymatic activity of Adh, the surrounding temperature, specific pre-treatments and the precise experimental procedure are responsible for this $32-34,39,58$. Our results also suggest that certain mutations may alter the attraction to ethanol, as $w^{1118}$ mutants showed a reduced attraction to higher ethanol concentrations compared to the wildtype (Fig. 1).

Substrate attraction tests (Fig. 1 and Fig. 2) allow larvae to come into direct contact with ethanol. Therefore, larvae can potentially be guided by different inputs: the sense of smell or taste, but also by a caloric gain or a pharmacological effect. Examples for such cases are reported for adult $D$. melanogaster (reviewed in ${ }^{26,51,60}$ ). Therefore, we

275 have tried to separate some of these inputs from each other. (Please note that the pharmacological effect can only be analyzed by behavioral changes and measurement of endogenous ethanol concentration in the larvae, which were not done in our study). To investigate whether larvae specifically perceive and behaviorally respond to ethanol as an odorant, we have filled the stimulus in containers that allow ethanol to evaporate, but prevent the larvae from directly contacting it and thus excludes feeding or ingesting it (Fig. 4). This has not been studied so far. Larval ethanol attraction for the odorant stimulation was clearly evident (Fig. 4), but reduced compared to the substrate attraction of ethanol (Fig. 1). The behavioral response is almost halved from a median of 0.61 (Fig. 1) to 0.33 (Fig. 4). This means that (1) larvae indeed do perceive ethanol via the olfactory signaling pathway and (2) larvae can perceive ethanol not only as an odor. Both have to be accurately identified in the future with a standardized behavioral assay which is now available.

\section{Ethanol is both nutritious and toxic for the larva}

Survival experiments, as we have shown in Fig. 3, are often used to analyze a positive life-prolonging effect (e.g. based on additional calories of sugar in a minimal diet), or to identify harmful effects that lead to an increased mortality of larvae (e.g. by adding bitter compounds to the food) ${ }^{18-20}$. Our results suggest that ethanol performs two opposing functions.. Below $8 \%$ the positive - most likely nutritional effect - increases larval survival on pure agarose; at high concentrations above $8 \%$ ethanol toxicity prevails (Fig. 3). Many early studies focused on the effects of ethanol on larval survival and increases were referred to as tolerance (reviewed in ${ }^{27}$ ). Nowadays tolerance usually stands for an individual's resistance to the intoxicating effects of ethanol, according to its importance for humans ${ }^{27}$. Similar to our results (Fig. 3), previous work 
showed that ethanol consumption of up to $4 \%$ leads to increased larval fitness $47,48,61,62$. Parson and colleagues showed that ethanol concentrations of $0.5 \%-3 \%$ allow larvae to develop to $3^{\text {rd }}$ instar larvae. At a concentration of $6 \%$ or higher this was not the case ${ }^{48}$. Quite similar, McKechnie and Geer showed that wild-type Canton-S larvae transferred to ethanol concentrations of up to $4.5 \%$ partially survived into pupae 47. Thus our data in line with published results suggest that Drosophila larvae may benefit from metabolizing ethanol. Since ethanol is one of the primary alcohols, it is degraded to aldehydes which then act as acetyl-CoA in metabolic processes such as the Krebs cycle (reviewed in ${ }^{43}$ ). Thus, ethanol contributes to the formation of 310 adenosine triphosphate (ATP) which can be used directly or indirectly as an energy source. Studies in larvae have additionally shown that ethanol's carbons serve as backbones for triacylglycerols and phospholipids 61,63 . Consequently, it can be assumed that larvae benefit from metabolizing the quantities of ethanol that they usually encounter in their natural environment.

At higher ethanol concentrations the Adh dependent detoxification reaches its limits. In most studies, reduced survival rates occur at concentrations above $9 \%$ or $16 \%$ of ethanol ${ }^{28,46,48}$ (but see also for lower concentrations ${ }^{47}$ ). Our results are therefore comparable with most of the published work, as we show that larvae do not display increased mortality at $8 \%$ ethanol, but many of them die quickly at an ethanol concentration of $20 \%$ (Fig. 3). It was shown that high intracellular concentrations of ethanol limits the capacities to synthesis and store glycogen, lipid and protein in the larval fat body cells. Even structural changes within cells of the endoplasmatic reticulum and mitochondria were visible ${ }^{61}$. Therefore, higher doses of ethanol have a deleterious effect at both the cellular and organism levels.

Surprisingly, larvae still seek out these deleterious concentration and process it as rewarding in learning experiments (Fig. 1, Fig. 3, Fig. 5 and Fig. 7). Thus, it is tempting to speculate that the answer is based on the organization of the larval microhabitats.

330 Ethanol occurs most often as a gradient in food sources. Therefore, it might be advantageous for the larva to seek out even high ethanol sources in the first step to then consume lower substrate concentrations on site in the second step. In general, however, it appears that larvae respond differently to a given concentration of a chemosensory stimulus depending on their behavioral response. $0.3 \mathrm{M}$ sodium chloride is usually repellent to larvae, but can be used as a positive teaching signal in olfactory learning experiments ${ }^{42}$. $2 \mathrm{M}$ arabinose, a sugar that is sweet but offers no nutritional benefit, is harmful for the larva, but also highly attractive and triggers a reward function during learning ${ }^{20}$. It appears that this is a general function for many chemosensory components, which may be based on mutual neuronal and genetic mechanisms. 


\section{Ethanol provides a positive teaching signal}

To our knowledge it has not yet been tested whether larvae can utilize ethanol as a positive teaching signal (unconditioned stimulus) in learning and memory assays. Our results suggest that this is the case for concentrations of $8 \%$ and $20 \%$ ethanol (Fig.

350 5). Therefore, not only adult flies can use ethanol as a teaching signal ${ }^{64,65}$, but also larvae. The initial memory of adult flies, however, is aversive and only changes after 24 hours into a positive long-term memory that lasts several days ${ }^{64}$. The adult behavioral response is therefore clearly different from the immediately positive larval response. Nonetheless, we gain increasing insight to understand what stimulation the larva classifies as rewarding. Chemosensory stimuli include ethanol, low salt concentrations, amino acids, ribonucleosides and various sugars (e.g., sucrose, glucose, maltodextrin, sorbitol, ribose), even though some cannot be metabolized (arabinose, xylose) 20,21,42,49,66,67.

360 We also do not yet have any insights into the neuronal and molecular organization of larval ethanol learning and memory. However, the here described assay now allows their analysis. It is worth noting that ethanol memory cannot be retrieved on a fructose test plate (Fig. 5). According to Schleyer and colleagues D. melanogaster larvae search for food after the conditioning phase based on their acquired experience. Thus,

365 the odorant paired with ethanol predicts a certain gain. At the moment of testing, the animals expect such a gain and compare it with their current environmental input. It seems that the larvae do not only expect a positive gain in this situation but also its specific quality. This was shown as sugar memory could be recalled on a positive aspartic acid containing test plate and vice versa ${ }^{68}$. Following the same logic, the 370 absence of ethanol memory recall on a fructose plate thus means that larvae do not distinguish between ethanol and fructose quality. Therefore, it is possible that fructose and ethanol processing circuits overlap in the larval brain.

\section{Outlook}

Which cells and molecules might be involved in the perception of ethanol in larvae? Based on studies on olfactory ethanol sensing in adult flies, the octopaminergic system and different olfactory receptors can be considered as an analysis entry point 69,70 . Using the Flywalk ${ }^{71}$ or a two odor vial assay ${ }^{72}$ to measure naive adult olfactory output, 380 it was shown that the olfactory co-receptor Orco plays an essential role in both assays when ethanol was applied. For the Flywalk analysis the effect could even be refined to the olfactory receptor genes Or42b and Or59b ${ }^{70}$. At the larval stage, only Or42b is expressed in a single ORN of the dorsal organ, the main larval olfactory sense organ 15. Or42b responds to ethyl acetate, ethyl butyrate, propyl acetate and pentyl acetate; chemicals that are all highly attractive to larvae ${ }^{16,17}$. It is tempting to expect that Or42b serves a similar function at the larval stage. However, ethanol has not yet been tested, and we lack any molecular and neuronal information on its perception in the larva. With respect to learning and memory, we have recently obtained a cellular 
understanding of the involved neuronal pathways of the larval brain. Four dopaminergic neurons of the primary protocerebral anterior medial (pPAM) cluster encode fructose dependent teaching signals and these neurons are directly connected to the mushroom body, the larval memory center ${ }^{9,73-76}$. Hence, it is now possible to test whether aspects of the ethanol reward learning and memory are also processed by the same neuronal mushroom body network. This would also reveal a conservation 395 of the functional patterns throughout development, since adult appetitive ethanol memory also requires dopaminergic cells and the mushroom body 64 .

\section{Methods}

\section{Fly strains}

Fly strains were kept on standardized cornmeal medium containing $1 \%$ ethanol at $25^{\circ} \mathrm{C}$ and $65 \%$ humidity under a $14: 10 \mathrm{~h}$ light:dark cycle. Adult flies were transferred to new food vials every $72 \mathrm{~h}$. Larvae were taken from food vials and briefly washed in tap water to remove food residues. Two fly strains wild-type ${ }^{\mathrm{CS}}$ and mutant $w^{1118}$ were used in the experiments. For experiments at specific time points during larval development, larvae with defined age were used. If not otherwise mentioned larval of

405 different age were used. For staging larvae, adult flies were allowed to lay eggs for six hours; larvae were then collected in a 12 h cycle starting $36 \mathrm{~h}$ after egg laying (AEL).

\section{Substrate choice}

For gustatory preference tests, Petri dishes (85 mm diameter; Greiner) were filled with a thin layer of $2.5 \%$ agarose substrate (VWR Life Science; type number: 97062-250).

410 After cooling, agarose was removed from half of the plate and re-filled with $2.5 \%$ agarose substrate containing different concentration of $99.8 \%$ ethanol $(1 \%, 2 \%, 4 \%$, $8 \%, 10 \%, 20 \%$ or $30 \%$ ethanol; CHEMSOLUTE®; type number: 2273.1000$)$. To keep an evaporation effect as low as possible, groups of 30 larvae of different ages were placed immediately after plate preparation (in the order of maximum 45 minutes) in

415 the middle of the Petri dish and allowed to crawl for 5 min (or up to $120 \mathrm{~min}$ ) at room temperature (RT) ${ }^{55}$. Then, larvae were counted on each side of the Petri dish. A preference index (Pref) was calculated by subtracting the number of larvae on the pure agarose side (\#nS) from the number of larvae on the side with a stimuli (\#S), divided by the total number of larvae (\#total): Pref $=(\# S-\# n S) / \#$ total. A positive Pref indicates attraction and a negative aversion.

\section{Olfactory attraction}

To test for olfactory attraction, Petri dishes (85 mm diameter; Greiner) were filled with a thin layer of $2.5 \%$ agarose substrate or with $2.5 \%$ agarose substrate with different concentration of ethanol $(5 \%, 8 \%, 10 \%$ and $20 \%)$. To test the olfactory stimuli of ethanol, either $8 \%$ ethanol, benzaldehyde (BA; Sigma-Aldrich, type number: 102213897; undiluted), amyl acetate (AM; Sigma-Aldrich, type number: 102172386); 
diluted 1:250 in paraffin oil), 1-octanol (1-Oct; Sigma-Aldrich, type number: 101858766 undiluted) or distilled water was filled into custom-made Teflon containers $(4.5 \mathrm{~mm}$ diameter) with perforated lids and placed on each side of the plate. Immediately after plate preparation, groups of 30 larvae were placed in the middle of the Petri dish and allowed to crawl for 5 min at RT. Then, larvae were counted on each side of the Petri dish. A preference index (Pref) was calculated by subtracting the number of larvae on the side without an odor (\#nO) from the number of larvae on the side with an odor $(\# \mathrm{O})$, divided by the total number of larvae (\#total): Pref $=(\# \mathrm{O}-\# \mathrm{nO}) /$ \#total. A positive

435 Pref indicates an attractiveness, a negative Pref represents an avoidance.

\section{Olfactory ethanol learning and memory}

All experiments were performed on Petri dishes filled with a thin layer of either $2.5 \%$ pure agarose or $2.5 \%$ agarose containing different concentrations of ethanol $(8 \%$ or 20\%), 0.01M fructose (Carl Roth®; type number: 4981.2) or 1.5M sodium chloride

440 (Carl Roth®; type number: 3957.2). As olfactory stimuli, we filled $10 \mu \mathrm{AM}$, and $10 \mu \mathrm{l}$ $\mathrm{BA}$, in custom-made Teflon containers $(4.5 \mathrm{~mm}$ diameter) with perforated lids. Immediately after plate and container preparation, groups of 30 larvae were placed in the middle of the Petri dish containing a pure agarose substrate. All experiments were conducted at RT. Larvae were exposed to AM and allowed to crawl for $5 \mathrm{~min}$. Then,

445 larvae were transferred to a new Petri dish containing a positive (ethanol, fructose) or negative $(\mathrm{NaCl})$ reinforcer and exposed to $\mathrm{BA}$ for $5 \mathrm{~min}$. After three training cycles, larvae were placed on a new Petri dish containing pure agarose substrate or agarose substrate containing $8 \%$ ethanol, $0.01 \mathrm{M}$ fructose, or $1.5 \mathrm{M}$ sodium chloride and exposed to AM and BA on opposite sides for $5 \mathrm{~min}$. Then, larvae were counted on

450 each side of the Petri dish. A second group of larvae was trained via a reciprocal training regime. For each group an independent olfactory preference index was calculated as described above. A performance index $(P I)$ was calculated by adding the Pref of the first training group (Pref1) to the Pref of the second training group (Pref2) and dividing them by the number of experimental groups (\#2): $P I=($ Pref1 +

455 Pref2) / \#2. A positive PI indicates an attractiveness; a negative PI represents an avoidance.

\section{Larval survival on ethanol}

To investigate larval survival in the presence of ethanol, 12 wild-type L2 stage larvae were placed in vials containing either $1 \%$ pure agarose substrate or $1 \%$ agarose 460 substrate plus different concentrations of ethanol of $4 \%, 8 \%$ or $20 \%$. Three drops of tap water were daily added to prevent larvae from dehydrating. Larvae that were alive and later the pupal stage were counted from day 1 to day 9 . The percentage of survival and pupation were calculated as follows: Percentage $=$ (number of living larvae or pupae / total number of larvae / pupae) x 100. For each condition 16 independent experimental groups were analyzed $(n=16)$. 


\section{Data analysis and visualization}

To test whether single groups are significantly different from zero, we used a onesample t-test. Further statistical analyses were conducted using distribution-specific 470 tests. For normally distributed data, we used either the paired T-test with equal variance or the one-way ANOVA followed by a TukeyHSD multiple comparison test for normal distributed data. For non-normal distributed data, we used Wilcoxon signed rank test or Kruskal-Wallis test followed by Dunn's multiple comparison test. All statistical analyses and data visualization were done with R (V 3.5.1) in RStudio by 475 using stats, dunn.test and ggplot2 package. Figure panels were edited with Adobe Illustrator CS5 (San Jose, CA, USA). The significance level of statistical tests was set to 0.05 ; the shown confidence interval (ci) is the $95 \%$ confidence interval of the data plots. Data are displayed as box plots with the median as the middle line, the box boundaries as $25 \%$ and $75 \%$ quantiles and the whiskers as 1.5 times the interquartile

480 range. Outliers are shown as dots directly above or below the box plots. Further details are documented in the Supplemental Data files.

\section{Acknowledgments}

The authors thank Wolf Huetteroth, Tilman Triphan, Bert Klagges and Astrid Rohwedder for fruitful discussions and comments on the paper. This work was 485 supported by grants from the German Research Foundation (DFG) to AST (TH1584/31, TH1584/6-1 and TH1584/7-1) and HS (HS675/10-1).

\section{Author contributions}

IS and AST outlined the manuscript. IS, MB, HS and AST developed the design of the methodology; IS, MB, NN, YS and JS conducted the experiments; IS prepared and

490 created the analysis and visualization of the data; IS, MB, HS and AST wrote and finalized the manuscript.

\section{Competing interests}

The authors declare no competing interests.

\section{References}

4951 Kandel, E. R., Schwartz, J. H., Jessell, T. M., Siegelbaum, S. A. \& Hudspeth, A. J. Principles of Neural Science. Vol. 5 (McGraw-Hill Education Ltd, 2012).

2 Gerber, B., Stocker, R. F., Tanimura, T. \& Thum, A. S. Smelling, tasting, learning: Drosophila as a study case. Results Probl Cell Differ 47, 139-185, doi:10.1007/400_2008_9 (2009).

5003 Vosshall, L. B. \& Stocker, R. F. Molecular architecture of smell and taste in Drosophila. Annu Rev Neurosci 30, 505-533, doi:10.1146/annurev.neuro.30.051606.094306 (2007).

4 Liman, E. R., Zhang, Y. V. \& Montell, C. Peripheral coding of taste. Neuron 81, 9841000, doi:10.1016/j.neuron.2014.02.022 (2014). 
5055 Dahanukar, A., Hallem, E. A. \& Carlson, J. R. Insect chemoreception. Curr Opin Neurobiol 15, 423-430, doi:10.1016/j.conb.2005.06.001 (2005).

$6 \mathrm{Li}, \mathrm{H}$. H. et al. A GAL4 driver resource for developmental and behavioral studies on the larval CNS of Drosophila. Cell Rep 8, 897-908, doi:10.1016/j.celrep.2014.06.065 (2014).

5107 Ohyama, T. et al. A multilevel multimodal circuit enhances action selection in Drosophila. Nature 520, 633-639, doi:10.1038/nature14297 (2015).

8 Berck, M. E. et al. The wiring diagram of a glomerular olfactory system. Elife 5, doi:10.7554/eLife.14859 (2016).

9 Eichler, K. et al. The complete connectome of a learning and memory centre in an

10 Miroschnikow, A. et al. Convergence of monosynaptic and polysynaptic sensory paths onto common motor outputs in a Drosophila feeding connectome. Elife 7, doi:10.7554/eLife.40247 (2018).

11 Pfeiffer, B. D. et al. Refinement of tools for targeted gene expression in Drosophila.

12 Diegelmann, S., Klagges, B., Michels, B., Schleyer, M. \& Gerber, B. Maggot learning and Synapsin function. J Exp Biol 216, 939-951, doi:10.1242/jeb.076208 (2013).

13 Gerber, B. \& Stocker, R. F. The Drosophila larva as a model for studying chemosensation and chemosensory learning: a review. Chem Senses 32, 65-89, doi:10.1093/chemse/bjl030 (2007).

14 Widmann, A., Eichler, K., Selcho, M., Thum, A. S. \& Pauls, D. Odor-taste learning in Drosophila larvae. J Insect Physiol 106, 47-54, doi:10.1016/j.jinsphys.2017.08.004 (2018).

15 Fishilevich, E. et al. Chemotaxis behavior mediated by single larval olfactory neurons

16 Hoare, D. J. et al. Modeling peripheral olfactory coding in Drosophila larvae. PLoS One 6, e22996, doi:10.1371/journal.pone.0022996 (2011).

17 Kreher, S. A., Mathew, D., Kim, J. \& Carlson, J. R. Translation of sensory input into behavioral output via an olfactory system. Neuron 59, 110-124, doi:10.1016/j.neuron.2008.06.010 (2008).

18 Apostolopoulou, A. A. et al. Caffeine Taste Signaling in Drosophila Larvae. Front Cell Neurosci 10, 193, doi:10.3389/fncel.2016.00193 (2016).

19 Apostolopoulou, A. A., Mazija, L., Wust, A. \& Thum, A. S. The neuronal and molecular basis of quinine-dependent bitter taste signaling in Drosophila larvae. Front Behav

20 Rohwedder, A. et al. Nutritional value-dependent and nutritional value-independent effects on Drosophila melanogaster larval behavior. Chem Senses 37, 711-721, doi:10.1093/chemse/bjs055 (2012).

21 Schipanski, A., Yarali, A., Niewalda, T. \& Gerber, B. Behavioral analyses of sugar processing in choice, feeding, and learning in larval Drosophila. Chem Senses 33, 563573, doi:10.1093/chemse/bjn024 (2008).

22 Kim, H., Choi, M. S., Kang, K. \& Kwon, J. Y. Behavioral Analysis of Bitter Taste Perception in Drosophila Larvae. Chem Senses 41, 85-94, doi:10.1093/chemse/bjv061 (2016).

55023 Apostolopoulou, A. A. et al. Composition of agarose substrate affects behavioral output of Drosophila larvae. Front Behav Neurosci 8, 11, doi:10.3389/fnbeh.2014.00011 (2014). 
24 Kudow, N., Kamikouchi, A. \& Tanimura, T. Softness sensing and learning in Drosophila larvae. J Exp Biol 222, doi:10.1242/jeb.196329 (2019).

Parsons, P. A. The Evolutionary Biology of Colonizing Species. (Cambridge Univ. Press, 1983).

26 Kaun, K. R., Devineni, A. V. \& Heberlein, U. Drosophila melanogaster as a model to study drug addiction. Hum Genet 131, 959-975, doi:10.1007/s00439-012-1146-6 (2012).

56027 Scholz, H., Ramond, J., Singh, C. M. \& Heberlein, U. Functional ethanol tolerance in Drosophila. Neuron 28, 261-271, doi:10.1016/s0896-6273(00)00101-x (2000).

28 David, J. R. \& Bocquet, C. Similarities and differences in latitudinal adaptation of two Drosophila sibling species. Nature 257, 588-590, doi:10.1038/257588a0 (1975).

29 McDonald, J. F. \& Avise, J. C. Evidence for the adaptive significance of enzyme activity levels: interspecific variation in alpha-GPDH and ADH in Drosophila. Biochem Genet 14, 347-355, doi:10.1007/BF00484773 (1976).

30 Milan, N. F., Kacsoh, B. Z. \& Schlenke, T. A. Alcohol consumption as self-medication against blood-borne parasites in the fruit fly. Curr Biol 22, 488-493, doi:10.1016/j.cub.2012.01.045 (2012).

57031 Parsons, P. A. Larval reaction to alcohol as an indicator of resource utilization differences between Drosophila melanogaster and Drosophila simulans. Oecologia 30 , 141-146 (1977).

32 Parsons, P. A. \& King, S. B. Ethanol larval discrimination between two Drosophila sibling species. Experientia 33, 898-899 (1977).

57533 Parsons, P. A. Larval Responses to Environmental Ethanol in Drosophila melanogaster: Variation Within and Among Populations. Behavior Genetics 10, 183190 (1980).

34 Cavener, D. Preference for ethanol in Drosophila melanogaster associated with the alcohol dehydrogenase polymorphism. Behav Genet 9, 359-365,

35 Oakeshott, J. G. et al. Alcohol Dehydrogenase and Glycerol-3-Phosphate Dehydrogenase Clines in Drosophila Melanogaster on Different Continents. Evolution 36, 86-96, doi:10.1111/j.1558-5646.1982.tb05013.x (1982).

36 David, J., Mercot, H., Capy, P., McEvey, S. \& Van Herrewege, J. Alcohol tolerance and Adh gene frequencies in European and African populations of Drosophila melanogaster. Genet Sel Evol 18, 405-416, doi:10.1186/1297-9686-18-4-405 (1986).

7 McKechnie, S. W. \& McKenzie, J. A. Polymorphism of Alcohol Dehydrogenase (Adh) in a Winery Cellar Population of Drosophila Melanogaster: Gene Frequency Association with Temperature and Genotypic Differences in Progeny Production. Evolution 37, 850-853, doi:10.1111/j.1558-5646.1983.tb05606.x (1983).

38 Cobb, M., Bruneau, S. \& Jallon, J. M. Genetic and developmental factors in the olfactory response of Drosophila melanogaster larvae to alcohols. Proc Biol Sci 248, 103-109, doi:10.1098/rspb.1992.0048 (1992).

39 Gelfand, L. J. \& McDonald, J. F. Relationship between ADH activity and behavioral response to environmental alcohol in Drosophila. Behav Genet 10, 237-249, doi:10.1007/BF01067770 (1980).

40 El-Keredy, A., Schleyer, M., Konig, C., Ekim, A. \& Gerber, B. Behavioural analyses of quinine processing in choice, feeding and learning of larval Drosophila. PLoS One 7, e40525, doi:10.1371/journal.pone.0040525 (2012). 
$60041 \quad$ Schleyer, M. et al. A behavior-based circuit model of how outcome expectations organize learned behavior in larval Drosophila. Learn Mem 18, 639-653, doi:10.1101/lm.2163411 (2011).

42 Niewalda, T. et al. Salt processing in larval Drosophila: choice, feeding, and learning shift from appetitive to aversive in a concentration-dependent way. Chem Senses 33,

605 685-692, doi:10.1093/chemse/bjn037 (2008).

43 Heinstra, P. W. H. Evolutionary genetics of the Drosophila alcohol dehydrogenase gene-enzyme system. Genetica 92, 1-22 (1993).

44 Libion-Mannaert, M., Delcour, J., Deltmobe-Lietaert, M. C., Lenelle-Montfort, N. \& Elens, A. Ethanol as a "Food" for Drosophila melanogaster: Influence of the Ebony Gene. Experientia 32, 22-24 (1976).

45 Pecsenye, K., Lefkovitch, L. P., Giles, B. E. \& Saura, A. Does Drosophila melanogaster use ethanol as an energy source during starvation? Hereditas 121, 225-236 (1994).

46 Fry, J. D. Direct and correlated responses to selection for larval ethanol tolerance in Drosophila melanogaster. J Evol Biol 14, 296-309 (2001).

61547 McKechnie, S. W. \& Geer, B. W. Regulation of Alcohol Dehydrogenase in Drosophila melanogaster by dietary Alcohol and Carbohydrate. Insect Biochem 14, 231-242 (1984).

48 Parson, P. A., Stanley, S. M. \& Spence, G. E. Environmental Ethanol at Low Concentrations: Longevity and Development in the Sibling Species Drosophilu melanogaster and D, simuluns. Aust J Zool 27, 747-754 (1979).

49 Gerber, B. \& Hendel, T. Outcome expectations drive learned behaviour in larval Drosophila. Proc Biol Sci 273, 2965-2968, doi:10.1098/rspb.2006.3673 (2006).

50 Robinson, B. G. et al. A low concentration of ethanol impairs learning but not motor and sensory behavior in Drosophila larvae. PLoS One 7, e37394, doi:10.1371/journal.pone.0037394 (2012).

51 Devineni, A. V. \& Heberlein, U. The evolution of Drosophila melanogaster as a model for alcohol research. Annu Rev Neurosci 36, 121-138, doi:10.1146/annurev-neuro062012-170256 (2013).

52 Guarnieri, D. J. \& Heberlein, U. Drosophila melanogaster, a genetic model system for alcohol research. International review of neurobiology 54, 199-228, doi:10.1016/s0074-7742(03)54006-5 (2003).

53 Dudley, R. Ethanol, fruit ripening, and the historical origins of human alcoholism in primate frugivory. Integr Comp Biol 44, 315-323, doi:10.1093/icb/44.4.315 (2004).

54 McKenzie, J. A. \& McKechnie, S. W. A comparative study of resource utilization in natural populations of Drosophila melanogaster and D. simulans. Oecologia 40, 299309, doi:10.1007/BF00345326 (1979).

55 Gibson, J. B., May, T. W. \& Wilks, A. V. Genetic Variation at the Alcohol Dehydrogenase Locus in Drosophila Melanogaster in Relation to Environmental Variation: Ethanol Levels in Breeding Sites and Allozyme Frequencies. Oecologia 51,

56 Stamps, J. A., Yang, L. H., Morales, V. M. \& Boundy-Mills, K. L. Drosophila regulate yeast density and increase yeast community similarity in a natural substrate. PLOS One 7, e42238, doi:10.1371/journal.pone.0042238 (2012).

57 Rodrigues, V. Olfactory behavior of Drosophila melanogaster. Basic Life Sci 16, 361371, doi:10.1007/978-1-4684-7968-3_26 (1980). 
58 Depiereux, E. et al. Larval Behavioral Response to Environmental Ethanol in Relation to Alcohol Dehydrogenase Activity Level in Drosophila melanogaster. Behavior Genetics 15, 181-188 (1985).

59 Gelfand, L. J. \& McDonald, J. F. Relationship between alcohol dehydrogenase (ADH) activity and behavioral resonse to environmental alcohol in five Drosophila species. Behavior Genetics 13, $281-293$ (1983).

60 Das, G., Lin, S. \& Waddell, S. Remembering Components of Food in Drosophila. Front Integr Neurosci 10, 4, doi:10.3389/fnint.2016.00004 (2016).

61 Geer, B. W., Dybas, L. K. \& Shanner, L. J. Alcohol dehydrogenase and ethanol tolerance at the cellular level in Drosophila melanogaster. J Exp Zool 250, 22-39, doi:10.1002/jez.1402500105 (1989).

62 McKenzie, J. A. \& Parsons, P. A. Alcohol tolerance: An ecological parameter in the relative success of Drosophila melanogaster and Drosophila simulans. Oecologia 10, 373-388, doi:10.1007/BF00345738 (1972).

66063 Geer, B. W., Langevin, M. L. \& McKechnie, S. W. Dietary Ethanol and Lipid Synthesis in Drosphila melanogaster. Biochemical Genetics 23, 607-622 (1985).

64 Kaun, K. R., Azanchi, R., Maung, Z., Hirsh, J. \& Heberlein, U. A Drosophila model for alcohol reward. Nat Neurosci 14, 612-619, doi:10.1038/nn.2805 (2011).

65 Nunez, K. M., Azanchi, R. \& Kaun, K. R. Cue-Induced Ethanol Seeking in Drosophila melanogaster Is Dose-Dependent. Front Physiol 9, 438, doi:10.3389/fphys.2018.00438 (2018).

66 Mishra, D., Thorne, N., Miyamoto, C., Jagge, C. \& Amrein, H. The taste of ribonucleosides: Novel macronutrients essential for larval growth are sensed by Drosophila gustatory receptor proteins. PLoS Biol 16, e2005570, doi:10.1371/journal.pbio.2005570 (2018).

67 Toshima, N., Kantar Weigelt, M., Weiglein, A., Boetzl, F. A. \& Gerber, B. An aminoacid mixture can be both rewarding and punishing to larval Drosophila melanogaster. J Exp Biol 222, doi:10.1242/jeb.209486 (2019).

68 Schleyer, M., Miura, D., Tanimura, T. \& Gerber, B. Learning the specific quality of taste

69 Schneider, A. et al. Neuronal basis of innate olfactory attraction to ethanol in Drosophila. PLoS One 7, e52007, doi:10.1371/journal.pone.0052007 (2012).

70 Keesey, I. W. et al. Alcohol boosts pheromone production in male flies and makes them sexier. doi:10.1101/2020.08.09.242784 (2020).

Steck, K. et al. A high-throughput behavioral paradigm for Drosophila olfaction - The Flywalk. Sci Rep 2, 361, doi:10.1038/srep00361 (2012).

72 Ogueta, M., Cibik, O., Eltrop, R., Schneider, A. \& Scholz, H. The influence of Adh function on ethanol preference and tolerance in adult Drosophila melanogaster. Chem Senses 35, 813-822, doi:10.1093/chemse/bjq084 (2010).

68573 Selcho, M., Pauls, D., Han, K. A., Stocker, R. F. \& Thum, A. S. The role of dopamine in Drosophila larval classical olfactory conditioning. PLoS One 4, e5897, doi:10.1371/journal.pone.0005897 (2009).

74 Rohwedder, A. et al. Four Individually Identified Paired Dopamine Neurons Signal Reward in Larval Drosophila. Curr Biol 26, 661-669, doi:10.1016/j.cub.2016.01.012 (2016).

75 Saumweber, T. et al. Functional architecture of reward learning in mushroom body extrinsic neurons of larval Drosophila. Nat Commun 9, 1104, doi:10.1038/s41467-01803130-1 (2018). 
76 Lyutova, R. et al. Reward signaling in a recurrent circuit of dopaminergic neurons and peptidergic Kenyon cells. Nat Commun 10, 3097, doi:10.1038/s41467-019-11092-1 (2019). 


\section{Figures}

a

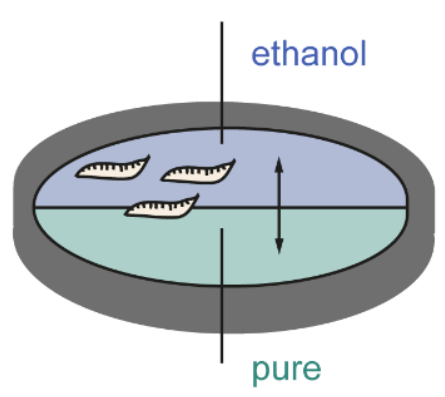

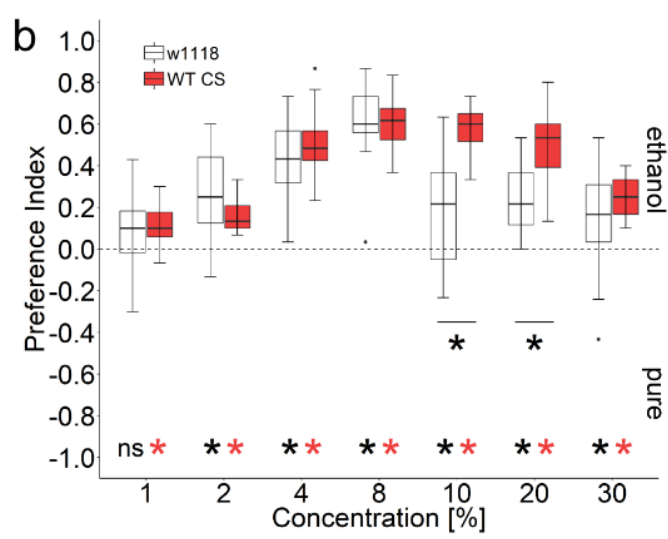

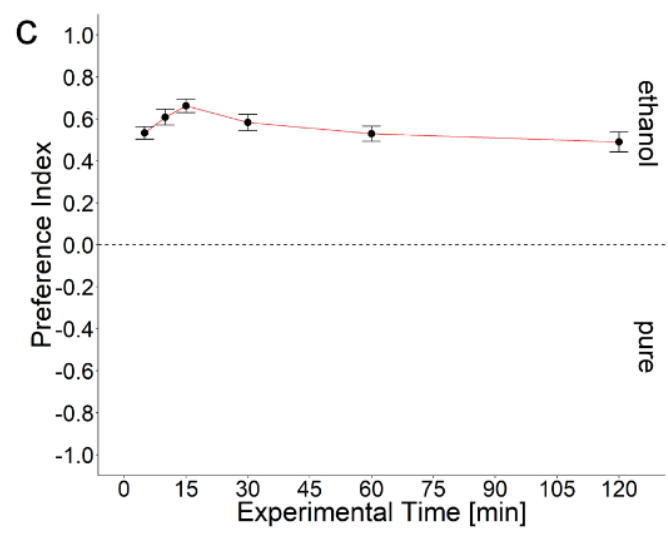

d 1.0

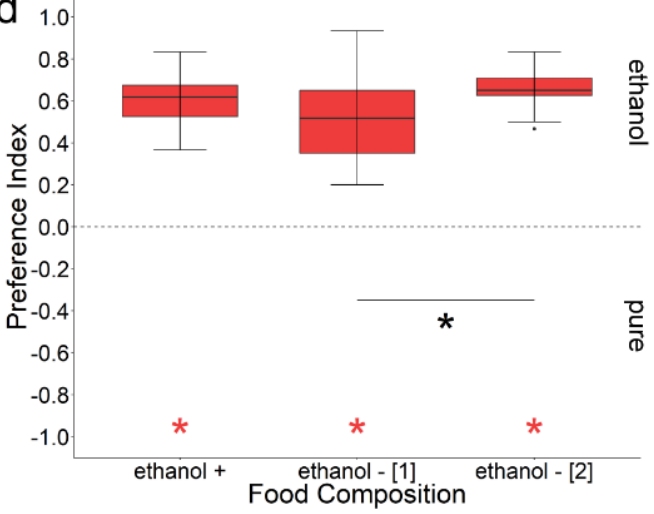

Figure 1: Ethanol attraction of Drosophila melanogaster larvae. (a) Scheme of the experimental procedure. Larvae were allowed to choose within five minutes between pure agarose and a substrate containing different concentrations of ethanol. (b) Wild-type Canton$S$ (red) and mutant $w^{1118}$ (white) larvae are attracted to ethanol. The highest behavioral response (preference index $($ Pref $)$ ) was seen at $8 \%$ ethanol for both strains (Pref ${ }^{\text {Canton-S }}=0.62$, $\left.\mathrm{ci}=0.53-0.68, \operatorname{Pref}^{v 1118}=0.60, \mathrm{ci}=0.51-0.74\right)$. All groups are significant different to zero, except for $1 \% w^{1118}$ (t-test, $p<0.17$ ). Multiple comparison indicates a significant difference between the two genotypes for $10 \%$ and $20 \%$ ethanol (Wilcoxon-Rank, $p^{10 \%}<0.001, p^{20 \%}<$

710 0.001). (c) Larval $8 \%$ ethanol substrate choice over a test period of 120 min. Canton-S larvae did not vary in their attraction within $120 \mathrm{~min}$ to $8 \%$ ethanol (Kruskal Wallis, $\mathrm{p}<0.032$ ). (Pref $f^{\mathrm{min}}$ $=0.55, \mathrm{ci}=0.47-0.6$, Pref $^{10 \mathrm{~min}}=0.65, \mathrm{ci}=0.53-0.69$, Pref $^{15 \mathrm{~min}}=0.63, \mathrm{ci}=0.6-0.73$, Pref $f^{30 \mathrm{~min}}$ $=0.53, \mathrm{ci}=0.5-0.67$, Pref $f^{60 \mathrm{~min}}=0.5, \mathrm{ci}=0.45-0.61$, Pref $\left.^{120 \mathrm{~min}}=0.57, \mathrm{ci}=0.39-0.59\right)$. Indicated values show medians; error bars represent standard errors. (d) Canton-S larvae raised on standard food that contains $1 \%$ ethanol (ethanol + ) shows a substrate choice behavior that was not different from larvae that were raised for one (ethanol - [1]) or two generations (ethanol - [2]) on ethanol free food (TukeyHSD, $p^{\text {standard-w/o }}<0.202, p^{\text {standarad-w/o2 }}<0.639$ ). Note a slight increase in ethanol substrate choice with increasing generations of ethanol food free raised flies (TukeyHSD, $p<0.03$ ). Differences against zero are indicated in red and black at the 
720 bottom of each panel. Sample size for each box plot is $n=16$. Significant differences of two groups are specifically indicated with an asterisk. Non-significant results are not indicated. Preference scores and statistical tests underlying the different indices are documented in the supplementary material.
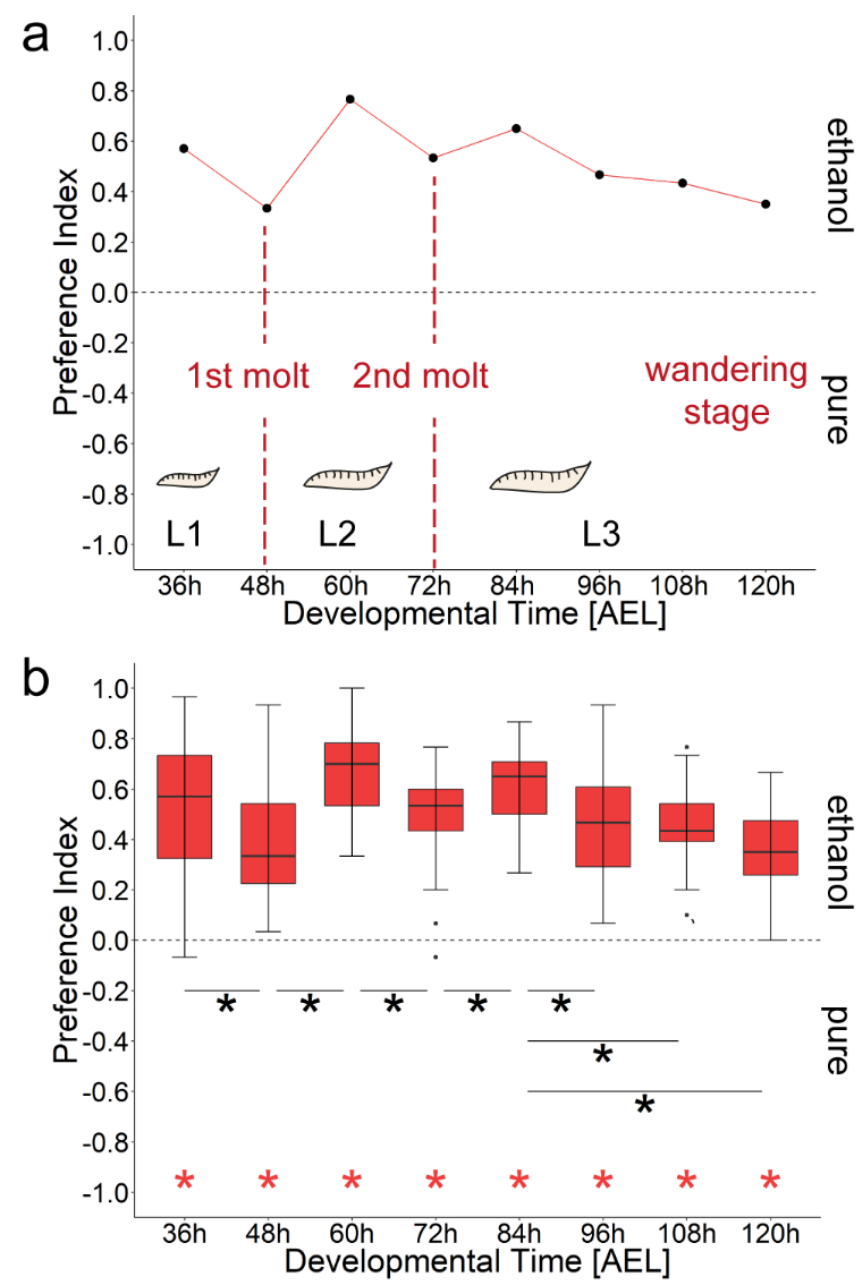

Figure 2: Ethanol substrate choice during larval development. (a) Canton-S larvae are attracted to $8 \%$ ethanol throughout the post-embryonal development. Data points represent the median behavioral response (substrate choice) according to the time after egg laying (AEL). Note a decrease of the behavioral response to some time points (48h AEL, 72h AEL, 96h-120h AEL) which correlates to larval molting events. (b) Corresponding box plots. Statistics are presented in Table 1. Differences from random choice are indicated in red at the bottom. Significant differences of two groups are specifically indicated with an asterisk. Nonsignificant results are not indicated. 
a
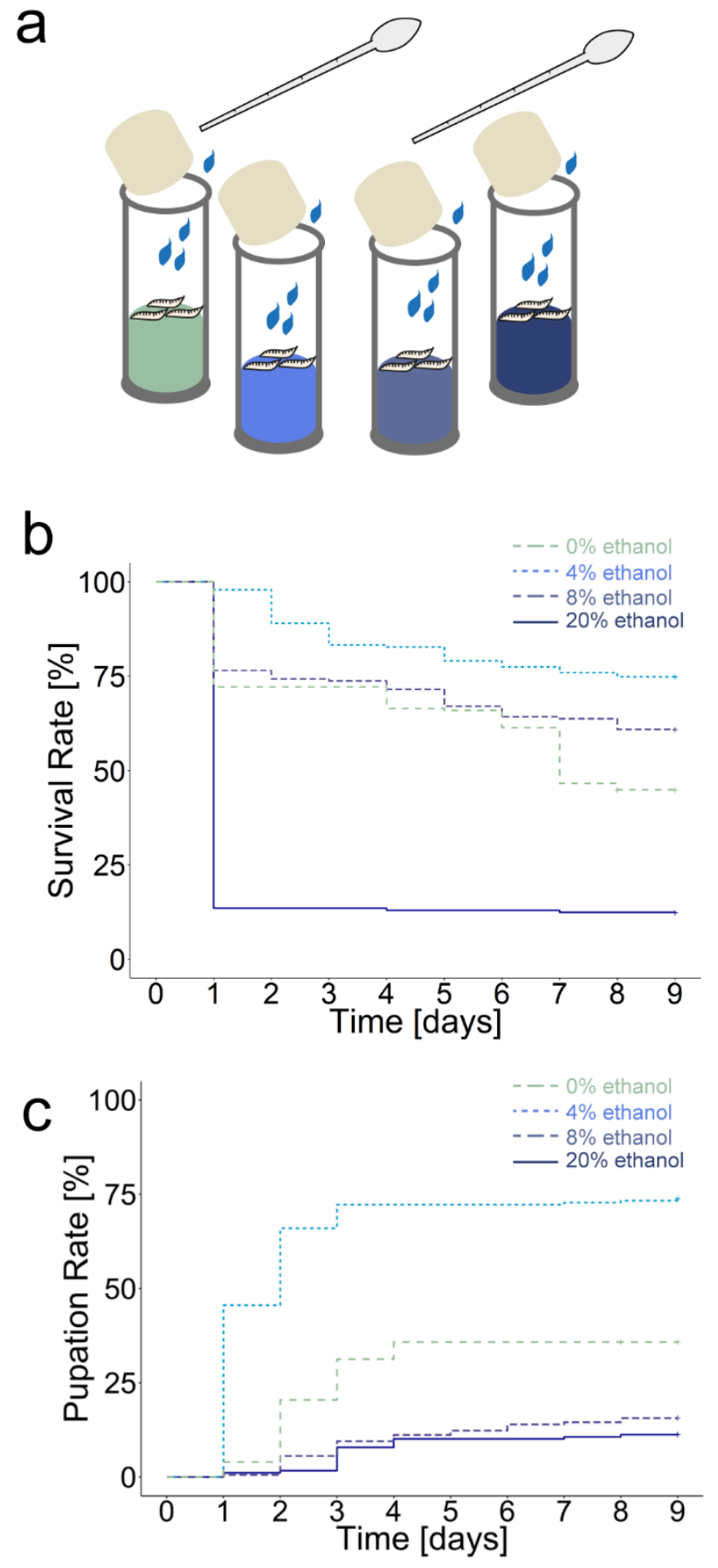

740 Figure 3: Larval survival on diets containing different concentrations of ethanol. (a) Scheme of the experimental procedure. Independent groups of 12 second instar Canton-S larvae were put into food vials that contained an agarose substrate plus different concentration of ethanol. Surviving of larvae and pupae were counted every $24 \mathrm{~h}$. Water was added every $24 \mathrm{~h}$ to avoid dehydration. Pupation was used as measure of survival. (b) Diagram shows 745 Kaplan-Meier survival curves of larvae. Larvae were put either on $0 \%$ (green), $4 \%$ (light blue), $8 \%$ (blue), or $20 \%$ ethanol (dark blue) diet for eight days. $20 \%$ ethanol significantly reduced larval survival as most of the animals died within one day (survival rate ${ }^{20 \% \text { Ethanol }}=12.40 \%$ ). Larvae reared on $0 \%$ and $8 \%$ ethanol showed a nearly similar survival (Log-Rank-test, $\mathrm{p}<$ 0.001 , survival rate ${ }^{0 \% \text { Ethanol }}=44.90 \%$, survival rate $e^{8 \% \text { Ethanol }}=60.90 \%$ ). In contrast $4 \%$ ethanol 
750 diet significantly increased larval survival compared to the $0 \%$ ethanol control diet (Log-Ranktest, $\mathrm{p}<0.001$, survival rate ${ }^{4 \% \text { Ethanol }}=74.90 \%$ ). (c) Diagram shows the related pupation rates of the surviving animals shown above raised at $0 \%$ (green), $4 \%$ (light blue), $8 \%$ (blue), or $20 \%$ ethanol (dark blue) diet. $4 \%$ ethanol diet significantly increased the pupation rate compared to larvae reared at $0 \%$ ethanol diet (Log-Rank-test, $p<0.001$, pupation rate ${ }^{0 \% \text { Ethanol }}=35.8 \%$, pupation rate ${ }^{4 \% \text { Ethanol }}=73.80 \%$ ). In contrast $8 \%$ and $20 \%$ ethanol diet significantly reduced the pupation rate compared to larvae reared at $0 \%$ ethanol diet (Log-Rank-test, $\mathrm{p}^{0 \%-8 \% \text { Ethanol }<}$ $0.001, \mathrm{p}^{0 \%-20 \% \text { Ethanol }}<0.001$, pupation rate ${ }^{8 \% \text { Ethanol }}=15.6 \%$, pupation rate $\left.e^{20 \% \text { Ethanol }}=12.2 \%\right)$. Single scores and statistical tests underlying the different survival curves are documented in the supplementary material. Sample size for each Kapplan-Meier survival curve is $n=16$.

a

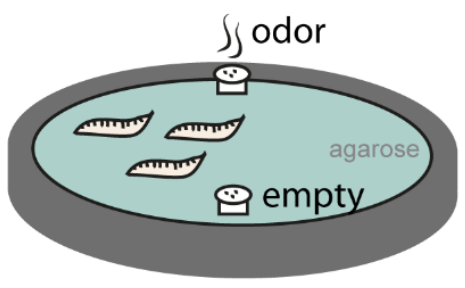

C

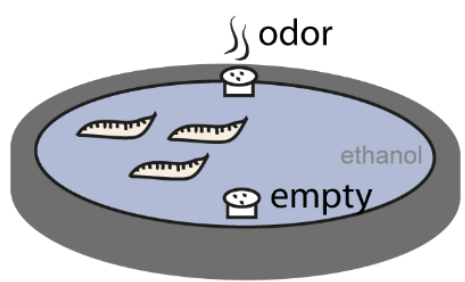

b

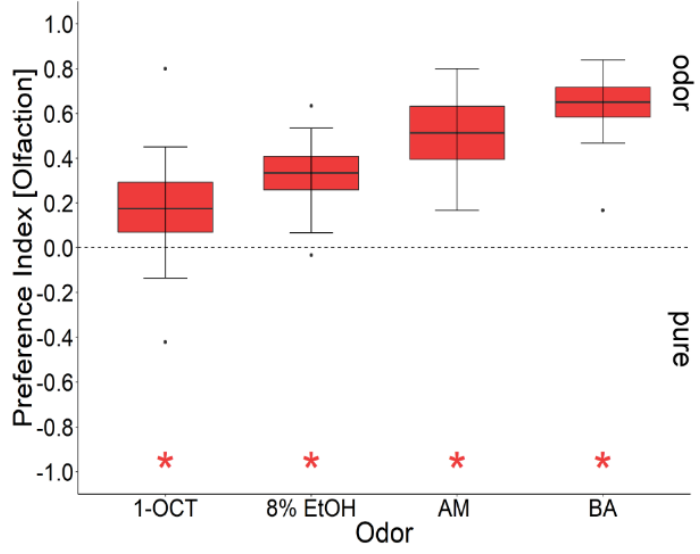

d

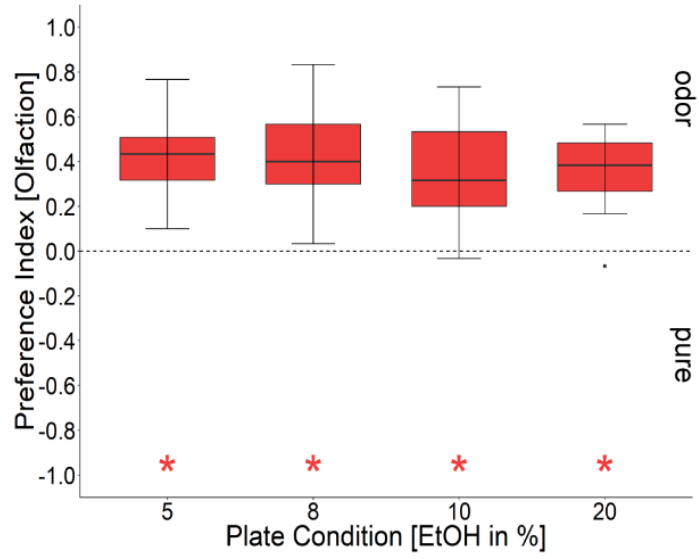

Figure 4: The effects of ethanol on larval olfactory attraction (a) Scheme of the experimental procedure. The olfactory stimuli 1-OCT, AM, BA, and $8 \%$ ethanol were presented on a $2.5 \%$ agarose plate in custom made Teflon containers to prevent direct contact of the animals with the chemical. Larvae were allowed to crawl for $5 \mathrm{~min}$. (b) Canton-S larvae preferred all odors including $8 \%$ ethanol over empty containers $\left(n^{1-O c t a n o l}=19\right.$, Pref ${ }^{1 \text {-Octanol }}=$ $0.17, \mathrm{ci}=0.04-0.29, \mathrm{n}^{\mathrm{AM}}=8, \operatorname{Pref}^{\mathrm{AM}}=0.51, \mathrm{ci}=0.3-0.69, \mathrm{n}^{8 \% \text { Ethanol }}=16$, Pre $f^{8 \% \text { Ethanol }}=0.33, \mathrm{ci}$ $\left.=0.24-0.41, \mathrm{n}^{\mathrm{BA}}=16, \operatorname{Pref}^{\mathrm{BA}}=0.65, \mathrm{ci}=0.55-0.72\right)$. All groups were significantly different to zero (t-test, $p^{1-\text { octanol }}<0.011, \mathrm{p}^{\mathrm{AM}}<0.001, p^{8 \% \text { Ethanol }}<0.001, p^{B A}<0.001$ ). (c) Scheme of the experimental procedure. Test plates contained $2.5 \%$ agarose mixed with either $5 \%, 8 \%, 10 \%$, or $20 \%$ ethanol. BA was presented in Teflon containers. Larvae were allowed to crawl for 5 
min. (d) Canton-S larvae showed an olfactory preference for BA irrespective of the ethanol concentrations in the test plate. All groups were significantly different to zero (t-test, $p^{B A-5 \% \text { Ethanol }}$ $\left.<0.001, p^{B A-8 \% \text { Ethanol }}<0.001, \mathrm{p}^{B A-10 \% \text { Ethanol }}<0.001, p^{B A-20 \% \text { Ethanol }}<0.001\right)$, but not different from each other (ANOVA, $p<0.697$ ). Sample size for each box plot is $n=16$. Differences against zero are indicated in red at the bottom of each panel. Significant differences of two groups are specifically indicated with an asterisk. Non-significant results are not indicated. Preference scores and statistical tests underlying the different indices are documented in the supplementary material.

a

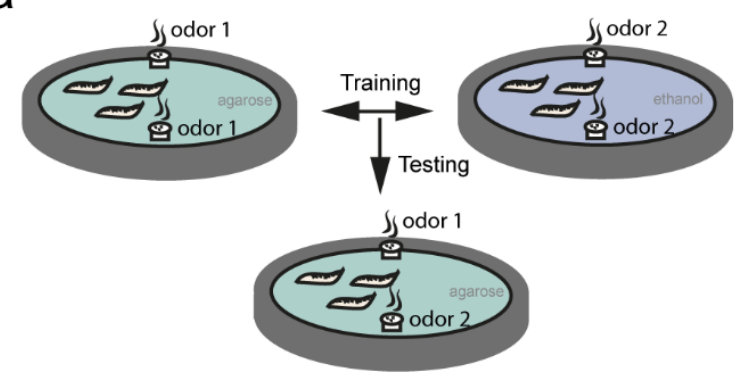

b

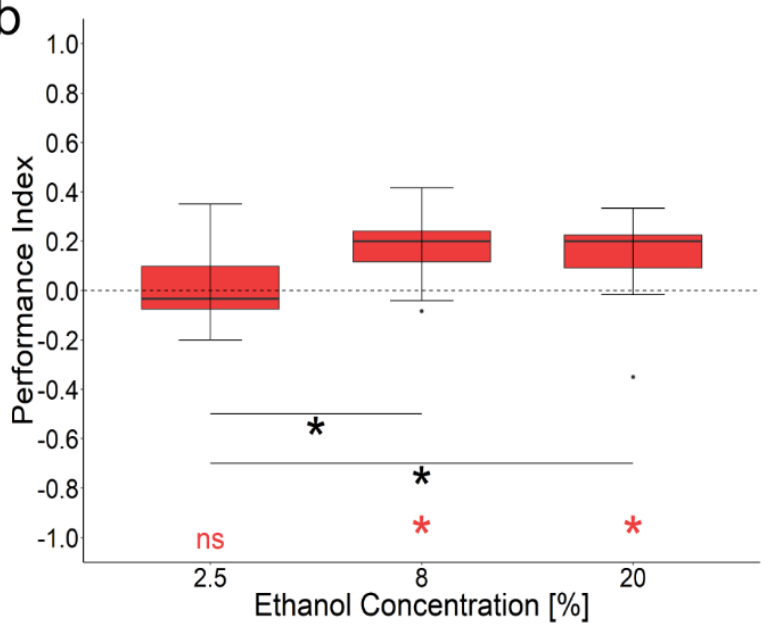

C

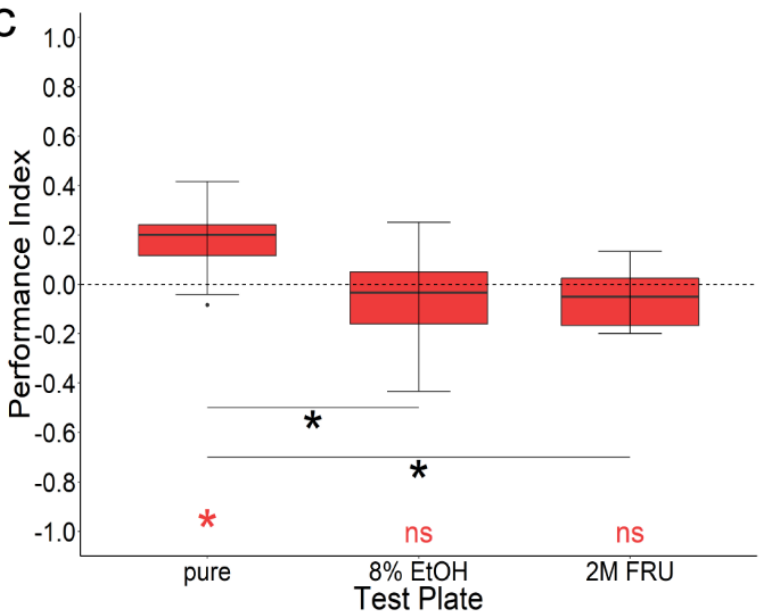

Figure 5: Larval olfactory learning reinforced by three different ethanol concentrations. (a) Scheme of the experimental procedure. Canton-S were trained three times with two odorants (AM and BA) and either $2.5 \%, 8 \%$, or $20 \%$ ethanol as reinforcer. Olfactory memory 
is quantified by the performance index (PI). (b) Larvae trained with $8 \%$ and $20 \%$ ethanol concentration show a significant appetitive memory $(P)^{8 \% \text { Ethanol }}=0.20, \mathrm{ci}=0.11-0.26$, $P P^{20 \% \text { Ethanol }}=0.20, \mathrm{ci}=0.05-0.24$, t-test, $\left.p^{8 \%}<0.001, p^{20 \%}<0.005\right)$. When $2.5 \%$ ethanol was used as a teaching signal no memory was detectable $(P)^{2.5 \% \text { thanol }}=0.83, \mathrm{ci}=-0.07-0.08$, $\mathrm{t}$ test, $p^{2.5 \%}<0.828$ ). Accordingly, larvae trained with $8 \%$ and $20 \%$ ethanol behaved differently from larvae trained with 2.5\% ethanol (Dunn's Multiple Comparison test, $p<0.001$ and $p<$ 0.003 , respectively). (c) Larvae trained with $8 \%$ ethanol concentration as teaching signal and tested on pure agarose showed a significant appetitive memory $(P I=0.20, \mathrm{ci}=0.11-0.26$, $\mathrm{t}$ test, $\mathrm{p}<0.001)$; if tested in the presence of the teaching signal of $8 \%$ ethanol or $2 \mathrm{M}$ fructose 795 (2M FRU), appetitive associative memory is not expressed $\left(P P^{\% \text { Ethanol }}=-0.03, \mathrm{ci}=-0.17-0.05\right.$, $\left.P^{2 M F r u}=-0.05, \mathrm{ci}=-0.11-0.01\right)$ as it is not significantly different from zero ( $\mathrm{t}$-test, $p^{8 \% \text { Ethanol }}<$ $0.260, p^{2 M F r u}<0.080$ ) and not significantly different from each other (paired t-test, $p<0.902$ ). Differences against zero are indicated in red at the bottom of each panel. Sample size for each box plot is $n=15$. Significant differences of two groups are specifically indicated with an asterisk. Preference scores and statistical tests underlying the different indices are documented in the supplementary material.
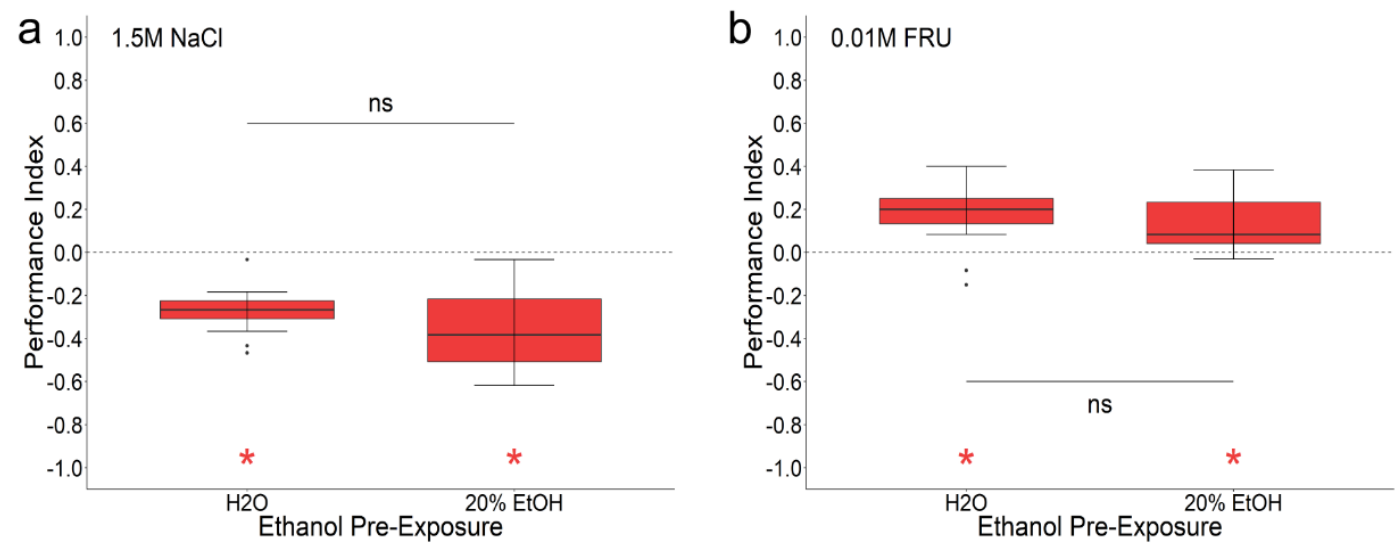

Figure 6: Pre-exposure to ethanol does not change aversive and appetitive olfactory learning. Before the larvae were conditioned, they were separated from their food and treated for 20 minutes with either water or $20 \%$ ethanol. They were then washed and trained with the odors $\mathrm{AM}$ and $\mathrm{BA}$ and either $1.5 \mathrm{M} \mathrm{NaCl}$ or $0.01 \mathrm{M}$ fructose as a teaching signal. (a) Larvae treated with water and $20 \%$ ethanol both showed an aversive odor-salt memory (t-test, pwater $<0.001$, ppre-EtOH $<0.001$ ) that was not different from each other (paired t-test, $p<0.112$ ). Sample size for each box plot is $n=15$. (b) Larvae treated with water and $20 \%$ ethanol both showed an appetitive odor-fructose memory (t-test, pwater $<0.001$, ppre-EtOH $<0.001$ ) that was not different from each other (paired t-test, $p<0.484$ ). Sample size for each box plot is $n$ $=15$. Differences against zero are indicated in red at the bottom of each panel. Significant differences of two groups are specifically indicated with an asterisk. Non-significant results are not indicated. Preference scores and statistical tests underlying the different indices are documented in the supplementary material. 

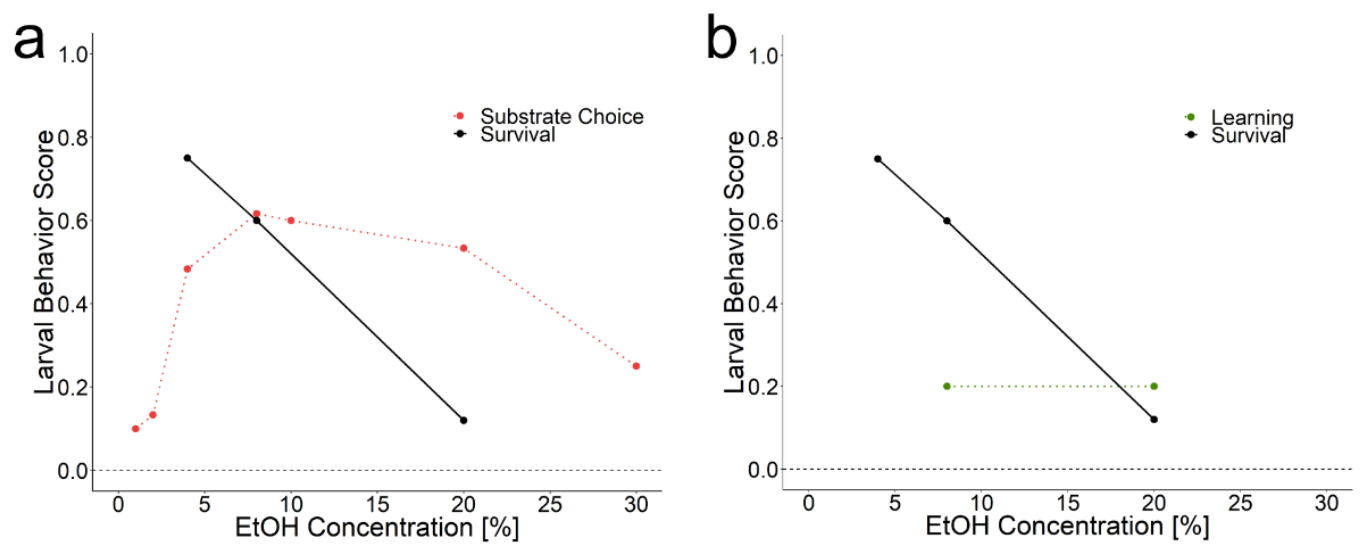

Figure 7: Summarizing semi schematic illustration of larval ethanol guided behavior scores. The $x$-axis shows ethanol concentrations from $0 \%$ to $30 \%$. The $y$-axis shows the larval behavior score from 0 to 1 for Canton-S. Note that the data plotted here are only comparable for the corresponding ethanol concentration. (a) Substrate choice ranges from 0.1 to 0.6 (data Fig. 1b). Survival scores indicates the survival probability at day eight (data Fig. 3b). Although higher concentrations of about $20 \%$ ethanol are harmful to the larvae a preference is still detectable. The behavioral curve for ethanol seems to be shifted towards higher concentrations. (b) Ethanol dependent memory indicates the performance scores for 8 and $20 \%$ ethanol (Fig. 5a). Survival scores indicate the survival probability at day eight (data Fig. $3 b)$. Although there is a clear negative effect on larval survival for higher concentrations of ethanol its rewarding effect stays the same. 
830 Table 1: Statistical analysis of substrate choice throughout the post-embryonal development of $D$. melanogaster.

\begin{tabular}{|c|c|c|c|c|c|}
\hline $\begin{array}{c}\text { Developmental } \\
\text { Time Points } \\
\text { (AEL) }\end{array}$ & $\mathbf{N}$ & $\begin{array}{l}\text { Preference } \\
\text { Index }\end{array}$ & $\begin{array}{l}\text { Statistics } \\
\text { Dunn's Multiple } \\
\text { Comparison }\end{array}$ & Event & $\mathrm{Cl}$ \\
\hline $36 h$ & 32 & 0.57 & \multirow{2}{*}{$p<0.001$} & \multirow{2}{*}{ 1st molt } & $0.44-0.63$ \\
\hline $48 h$ & 24 & 0.33 & & & $0.30-0.52$ \\
\hline $60 \mathrm{~h}$ & 31 & 0.77 & \multirow{2}{*}{$p<0.002$} & \multirow{2}{*}{ 2nd molt } & $0.59-0.73$ \\
\hline $72 h$ & 33 & 0.53 & & & $0.41-0.55$ \\
\hline $84 h$ & 16 & 0.65 & \multirow{2}{*}{$p<0.001$} & \multirow[t]{2}{*}{ Feeding L3 } & $0.51-0.69$ \\
\hline $96 h$ & 32 & 0.47 & & & $0.38-0.53$ \\
\hline $108 h$ & 16 & 0.43 & $\begin{array}{c}{ }^{*} \\
\text { Comparison } 84 \mathrm{~h} \\
\text { AEL } \\
p<0.017\end{array}$ & \multirow[t]{2}{*}{$\begin{array}{l}\text { Wandering } \\
\text { L3 }\end{array}$} & $0.35-0.54$ \\
\hline 120h & 16 & 0.35 & $\begin{array}{c}{ }^{*} \\
\text { Comparison } 84 \mathrm{~h} \\
\mathrm{AEL} \\
p<0.001\end{array}$ & & $0.27-0.45$ \\
\hline
\end{tabular}

